\title{
Antonymie et exhaustivité
}

\author{
Marie Steffens \\ Université de Liège, Belgique \\ Marie.Steffens@ulg.ac.be
}

Résumé. Seules de rares études se sont penchées sur la coprésence d'antonymes conventionnels en discours. Parmi celles-ci, celle de Steven Jones (2002) sur les fonctions discursives de la coprésence antonymique est devenue une référence. Notre communication se concentrera sur une fonction en particulier: l'antonymie coordonnée, sous-tendue par des structures syntaxiques tels que $X$ et / ou $Y$, où $\mathrm{X}$ et $\mathrm{Y}$ sont des antonymes. Grâce à cette fonction, la coprésence des antonymes peut exprimer l'exhaustivité, c'est-à-dire la totalité d'une dimension sémantique, selon Jones (Jones 2002 : 66). À partir de notre corpus francophone (Le Monde 1987-2002 et 2010-2011), nous avons extrait des exemples de coprésences antonymiques qui expriment l'exhaustivité lorsque les antonymes sont employés dans des structures syntaxiques identifiées par Jones, mais aussi au sein de structures syntaxiques non associées à l'antonymie coordonnée comme des comparaisons d'égalité : «Les absences de Federer sont aussi intenses que sa présence. » (Le Monde 02/06/2011). Notre communication visera à répondre aux deux questions suivantes : 1) Quelles sont les structures syntaxiques qui permettent aux antonymes d'exprimer l'exhaustivité ?; 2) Comment définir sémantiquement et référentiellement l'exhaustivité ? Pour répondre à la première question, nous présenterons une analyse des conditions syntaxiques et sémantiques dans lesquelles les antonymes expriment l'exhaustivité. Cette analyse sera fondée sur la méthode de description des relations entre prédicats et arguments proposée par Gaston Gross (2012) et utile pour décrire les relations entre les antonymes et les autres éléments de leur cotexte. Nous envisagerons ensuite l'exhaustivité comme l'expression d'une classe référentielle qui transcende, selon une dimension sémantique donnée (voir Mettinger 1994), la différence entre les deux classes référentielles associées aux antonymes.

\begin{abstract}
Only a few studies are dedicated to the copresence of conventional antonyms. Among these studies, the corpus-based description of discourse functions fulfilled through antonyms, made by Steven Jones (2002), became a reference. We will focus our communication on one function in particular: the coordinated antonymy, supported by syntactic frames such as $\boldsymbol{X}$ and/or $\boldsymbol{Y}$, where $\mathrm{X}$ and $\mathrm{Y}$ are antonyms. Through this function, the antonymic copresence can express exhaustiveness, that is to say all points of a semantic scale, according to Jones (2002:66). By searching into a French corpus constituted by articles from Le Monde (1987-2002 and 2010-2011), we found examples of antonyms which express exhaustiveness within syntactic frames similar to those identified by Jones, but also within syntactic frames not associated with coordinated antonymy, like equality comparisons, for example : « Les absences de Federer sont aussi intenses que sa présence. " (Le Monde 02/06/2011). In light of these examples, two questions arise: 1) What are the syntactic structures that allow antonyms to express completeness?; 2) How to define semantically and referentially the exhaustiveness expressed by antonyms in copresence? To answer the first question, we will present an analysis of syntactic and semantic conditions in which antonyms express completeness. This analysis will be based on the method of describing the relationship between predicates and arguments proposed by Gaston Gross (2012), and useful for describing the relationship between antonyms and other elements of their co-text. To answer the second question, we will describe antonyms as lexemes who designate referential prototypical categories. In this perspective, exhaustiveness subsumes the difference between two categories designated by antonyms to express a totality in regard to a semantic dimension (see Mettinger 1994).
\end{abstract}




\section{Antonymie coordonnée et exhaustivité}

Depuis Aristote, de nombreux travaux ont été consacrés à l'antonymie, conçue comme la relation entre des «mots qui, par le sens, s’opposent directement l'un à l'autre » (Grevisse 2003 : 31). L'étude de l'ancrage contextuel des associations antonymiques prend son essor à la suite d'études lexicométriques qui mettent en évidence l'importance quantitative des cooccurrences antonymiques, c'est-à-dire des coprésences ou présences simultanées de deux antonymes dans le même contexte linguistique (paragraphe, phrase ou fenêtre d'analyse arbitraire) dont la régularité est mesurée par la statistique (Mayaffre 2008 : 55). Les rares études, consacrées à ces coprésences et fondées sur l'analyse empirique de corpus de données textuelles, envisagent l'antonymie discursive sous deux aspects : 1) les structures syntaxiques, appelées patrons syntaxiques, qui sous-tendent la coprésence des antonymes en discours et 2) les « fonctions discursives » que leur mise en relation peut remplir.

Pour l'anglais, les patrons syntaxiques ont été décrits, à partir d'un corpus littéraire, par Arthur Mettinger (1994). Ces patrons sont repris et enrichis principalement par Steven Jones (2002) et adaptés au français par François Morlane-Hondère (2008). Le second aspect a été développé essentiellement par Jones (2002) et Jones et al. (2012) qui classent les coprésences antonymiques en dix catégories, fondées sur les différentes «fonctions discursives" (discourse functions) qu'elles peuvent exercer. À chaque «fonction » sont associés des patrons syntaxiques privilégiés. Selon Jones, l'une des fonctions le plus souvent excercées par les antonymes coprésents est l'antonymie coordonnée (coordinated antonymy): cette catégorie est illustrée par des phrases comme While pensions will not be abolished, the government will encourage everyone, rich and poor, to rely for their retirement mainly on money they invest in private pension funds (Jones 2002 : 61). Dans ce genre d'énoncé, les antonymes (X, Y) sont coordonnés par des structures syntaxiques du type $X$ or $Y, X$ and $Y$, ou juxtaposés sans conjonction de coordination (ibid. : 73-74, Jones et al. 2012 : 31). Le syntagme coordonnant exprime l'exhaustivité (exhaustiveness) (Jones 2002 : 61), c'est-à-dire « all points on the given scale, not just the two specific points mentioned » (ibid. : 66). L’énoncé ainsi construit peut renvoyer, selon Jones, à la totalité de l'échelle entre les antonymes, pôles et valeurs intermédiaires compris (ici, tout le monde, toute personne indépendamment de sa santé financière) (ibid. : 66-67).

Dans la conception de Jones, l'expression de l'exhaustivité est très liée à l'emploi d'une mise en relation des antonymes du type $X$ connecteur $Y$, où le connecteur exprime une coordination, c'est-à-dire où le connecteur est soit et, soit ou non exclusif (antonymie coordonnée). Cette conception tend à confondre la fonction des antonymes coprésents (expression de l'exhaustivité) et les structures syntaxiques qui soustendent cette coprésence (coordination). Ceci est problématique dans la mesure où, nous le verrons, il n’y a pas nécessairement de correspondance stricte entre les deux.

Grâce à l'analyse d'un corpus de textes écrits en français, nous cherchons à 1) distinguer les fonctions de la coprésence antonymique et les procédés formels sur lesquels elles s’appuient, 2) décrire les mécanismes sémantiques et syntaxiques de mise en relation contextuelle de deux antonymes, 3) définir la fonction sémantico-référentielle d'expression de l'exhaustivité par la coprésence d'antonymes en français.

Pour remplir ces objectifs et grâce à un moteur de recherche CQP, nous avons extrait d'un corpus, de plus de 500 millions de mots, composé d'articles du journal Le Monde (1987-2006, 2009-2011) des phrases (ou groupes de phrases) dans lesquelles deux antonymes sont employés ensemble. La liste des paires antonymiques à extraire a été établie à partir des renvois antonymiques du Grand Robert (2001). 


\section{Cadre d'analyse}

\subsection{La dimension sémantique}

La définition que donne Jones de l'exhaustivité comme l'expression de la totalité d'une échelle sémantique pose problème car elle ne peut s'appliquer aux cas où la coprésence d'antonymes non scalaires permet d'exprimer l'exhaustivité. Ainsi dans l'exemple (1), tiré de notre corpus, si les antonymes jeune/âgé, riche/pauvre, dépensier/avare et stable/précaire sont scalaires, c'est-à-dire qu'ils expriment les degrés d'une propriété et constituent les pôles d'une échelle sémantique, ce n’est pas le cas de homme/femme et de nomade/sédentaire.

(1) «Après une période d'observation continue des mouvements d'un téléphone, le moteur d'intelligence artificielle saura si son propriétaire est un homme ou une femme, jeune ou âgé, riche ou pauvre, dépensier ou avare, diplômé ou non, nomade ou sédentaire, employé stable ou précaire... » (Le Monde 11/05/2010, « Le téléphone qui en savait trop »,Yves Eudes).

L’exhaustivité ne doit donc pas être conçue comme la totalité d’une échelle sémantique mais comme la totalité d'une dimension sémantique. La dimension est définie, par Coseriu, comme « le point de vue ou critère d'une opposition donnée quelconque, c’est-à-dire, dans le cas d'une opposition lexématique, la propriété sémantique visée par cette opposition [...]» (Coseriu 1975 : 35) Elle est constituée de « ce qui est commun aux différences entre ces termes, c'est-à-dire à leurs traits distinctifs » (ibid. : 36). La dimension fournit le point commun sémantique par rapport auquel l'opposition entre deux antonymes est définie. Cette similarité entre les antonymes est nécessaire à leur opposition, comme à toute opposition : «[...] toute opposition a besoin d'un fond commun » (Blank 2000 : 61).

Ainsi dans l'énoncé (1), la coprésence antonymique permet d'exprimer que le téléphone pourra tout connaître sur son propriétaire, son sexe, son âge, sa fortune, son rapport à l'argent, à quelle fréquence il déménage et le statut de son emploi.

L'existence d'une dimension, nécessaire à l’opposition, est, par corollaire, présupposée par toute opposition entre des antonymes et, par conséquent, inférée dans le processus d’interprétation des coprésences antonymiques. Ainsi, quand la mise en contraste de deux antonymes permet la mise en contraste de deux autres lexèmes, une dimension d'opposition entre femmes et chiens est construite par l’interprétation :

(2) «Sa haine des femmes devint aussi légendaire que son amour pour les chiens : il appela son caniche Atma (les brahmanes désignent ainsi l'âme du monde), mais les habitants de la bonne ville de Francfort, où il mourut à l'âge de soixante-douze ans, le surnommaient Schopenhauer Junior. » (Le Monde 19/08/1988, « Portrait d’Arthur Schopenhauer, le rentier du pessimisme », Roland Jaccard).

\subsection{Relations prédicat-argument}

En ce qui concerne l'expression de l'exhaustivité, le modèle des patrons syntaxiques pour rendre compte des procédés de mise en relation de deux antonymes soulève deux remarques.

La première est que toutes les coordinations n'expriment pas l'exhaustivité comme le montrent les deux exemples suivants :

(3) «Ses ingénieurs [du DCNS] ont aussi retenu l'énergie thermique des mers, qui utilise les échanges de température entre l'eau chaude de la surface et l'eau froide remontant des profondeurs. » (Le Monde 16/04/2010, « DCNS veut prendre la tête d'une filière industrielle des énergies marines », Jean-Michel Bezat). 
(4) «Eliette Abécassis, dont je ne partage pas l'approche de la maternité, dit une chose juste : il y a deux sortes de femmes, celles qui aiment à se retrouver en femelles mammifères, et celles qui détestent cela, ne veulent pas en entendre parler. » ( $L e$ Monde 13/02/2010, «Cessons d'avoir une idée unique de la gent féminine », propos d’Elisabeth Badinter recueillis par Josyane Savigneau).

La deuxième est que l'exhaustivité peut être exprimée par d'autres structures syntaxiques et notamment celles qui sous-tendent la comparaison comme dans l'exemple suivant :

(5) «Les absences de Federer sont aussi intenses que sa présence. » (Le Monde 02/06/2011, “En 2 D ou en 3 D, le vent reste invisible”, André Scala).

Limiter l'analyse des coprésences antonymiques à l'identification des patrons syntaxiques qui les soustendent n'est donc pas pleinement satisfaisant. Pour analyser les relations entre les antonymes coprésents et leur environnement linguistique dans un énoncé donné, il faut décrire leur combinatoire. Pour ce faire, nous avons choisi d'adopter l'approche proposée par Mejri (2011) et Gross (2012).

Selon cette approche, trois grandes catégories de lexèmes peuvent être distinguées en regard des trois fonctions primaires que leur combinatoire leur permet d'exercer. Ces fonctions sont appelées fonction prédicative, fonction argumentale et fonction d'actualisation. Elles sont dites primaires parce que les composantes qui les exercent sont essentielles à la constitution de toute phrase : «[...] pour qu'il y ait phrase (énoncé), il faut qu’il y ait une relation entre des entités, inscrite dans le temps. » (Mejri 2011 : 10).

Seuls certains lexèmes, les lexèmes prédicatifs ou prédicats, peuvent exercer une fonction prédicative, ce sont ceux dont la combinatoire présuppose l'existence d'autres lexèmes, les arguments, dont leur signification dépend. À la suite de Zellig Harris (1976), Gross définit un lexème prédicatif comme « un mot qui opère une sélection déterminée parmi les mots du lexique pour établir avec eux un schéma formant la base d'une assertion " (Gross 2012 : 13). Les lexèmes prédicatifs peuvent être des verbes comme respecter dans Cet homme respecte les autres (ibid. : 17), des substantifs comme respect dans Cet homme a le respect des autres (ibid.), des adjectifs comme respectueux dans Cet homme est respectueux des autres (ibid. : 18), des prépositions comme contre dans Cet homme est contre les autres (ibid.) ou des adverbes comme sérieusement dans Paul travaille sérieusement (ibid. : 287). Les lexèmes prédicatifs peuvent également être sélectionnés par d'autres lexèmes prédicatifs pour exercer une fonction argumentale par rapport à eux. Selon cette définition, les antonymes du français sont pour l'essentiel des lexèmes prédicatifs.

Dans un énoncé comme Hier, Jean a offert des fleurs à Marie, a offert exerce une fonction prédicative, Jean, des fleurs et Marie exercent une fonction argumentale parce qu'ils sont sélectionnés par le prédicat et hier exerce une fonction d'actualisation parce qu'il participe « au passage de l'existence virtuelle de la relation prédicative à son existence actuelle, c'est-à-dire celle qui est prise en charge par un locuteur qui l'inscrit dans le temps et l'espace. » (Mejri 2011 : 10-11).

\subsection{Simple mise en relation et double mise en relation}

À côté de l'antonymie coordonnée, Jones (2002) met en évidence la grande fréquence d'emploi simultané, dans un énoncé donné, des deux membres d'une paire antonymique (paire A) pour appuyer, voire rendre possible, la mise en relation contrastive d'autres unités ou groupes d'unités présents dans le même énoncé (paire B). Dans ces cas, Jones considère que la coprésence antonymique se met au service de la mise en relation des membres de la paire B et parle d'antonymie ancillaire (ancillary antonymy) (Jones 2002 : 45).

Pour identifier ce phénomène et le décrire dans nos exemples en français, en-dehors du cadre théorique dans lequel le place Jones, nous lui donnerons le nom de double mise en relation. Il y a double mise en relation lorsque la coprésence antonymique participe à la mise en relation d'autres lexèmes du cotexte, la paire B, comme c'est le cas dans l'exemple (2), par opposition aux cas dans lesquels aucune paire B ne 
peut être identifiée. Nous dirons, dans ces cas, que les antonymes coprésents participent à une simple mise en relation, la leur. Pour distinguer la double mise en relation et la simple mise en relation, il faut définir les critères qui permettent de déterminer si une paire B peut être identifiée.

D’après les énoncés relevés par Jones et nos propres analyses, nous pouvons définir ce critère d'identification d'une paire $B$ comme suit : dans un énoncé où deux antonymes sont coprésents, il y a une paire B si les antonymes exercent, directement ou indirectement (i.e. par le biais d'une relation de dépendance avec un autre prédicat), une fonction prédicative, argumentale ou d'actualisation, de même rang, par rapport à des lexèmes ou syntagmes qui désignent des référents différents.

Selon ce critère, la paire B femmes/chiens peut être identifiée clairement dans l’énoncé (2), ci-dessus.

(6) «Tout en riant et pleurant avec les héros, nous plongeons dans la vie quotidienne d'un hameau - avec sa maîtresse d'école, son médecin, son diacre, ses religieuses, ses bachi-bouzouks -, vivant ses dernières heures. » (Monde 07/09/2007, « Ivan Vazov, naissance d'une nation », Marie Zawisza).

Dans l'énoncé (6), à l’inverse, les lexèmes prédicatifs antonymes ont le même argument, nous. Les actions dénotées par ces verbes sont accomplies par le même groupe de personnes. Aucune paire B ne peut donc être identifiée.

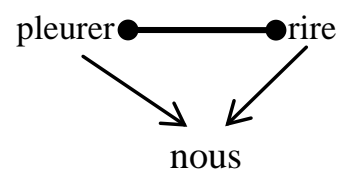

L’application de ce critère d’identification est plus délicate dans l’énoncé ci-dessous.

(7) «Confortable pour un film plutôt documentaire, qui renvoie à la vie, aux photos et aux films de Depardon, qui mêle des images anciennes et récentes, animées et fixes. » (Le Monde 14/05/2011, « Raymond Depardon tourne un film à Cannes », Michel Guerrin).

Dans cet énoncé, les membres des deux paires d'adjectifs antonymes semblent avoir le même argument, images. Or, il est important de remarquer que, en raison de la présence des antonymes prédicatifs, images en tant qu'argument de ancien (ou animé) ne peut désigner les mêmes référents que images en tant qu'argument de récent (ou fixe). Le lexème images n'étant exprimé qu'une fois, il faut réactiver, pour l'intreprétation d'un tel énoncé, un lexème sous-entendu qui ne renvoie pas à la même classe référentielle que le lexème exprimé, ce qui est noté en grisé dans les schémas ci-dessous. Dans ce cas, contrairement à l'énoncé (6), on peut considérer qu'il y a bel et bien une paire B.
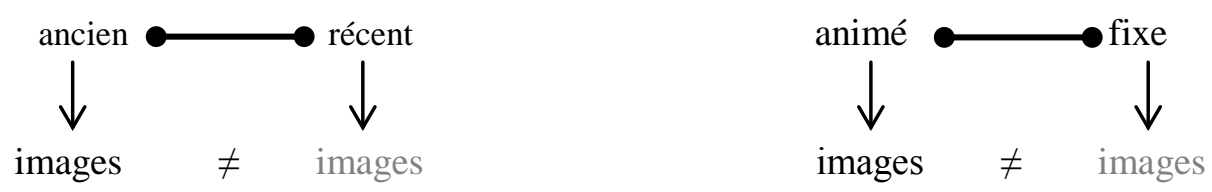

\section{Expression de l'exhaustivité}

La coprésence antonymique peut exprimer l'exhaustivité dans deux configurations différentes, lorsque les antonymes participent à une simple mise en relation (exhaustivité simple) et lorsque les antonymes participent à une double mise en relation (exhaustivité par rétroclassement). Nous allons présenter ces deux configurations. 


\subsection{Exhaustivité simple}

Lorsque des antonymes coprésents expriment l'exhaustivité dans une simple mise en relation, ils peuvent occuper la même position argumentale par rapport à un prédicat, en être les actualisateurs ou être les prédicats recteurs des propositions dans lesquelles ils s’insèrent. Ils ont alors tous leurs arguments en commun. Nous allons envisager ce cas de figure en premier lieu.

\subsubsection{Antonymes dans une fonction prédicative}

$A_{1}(C) . A_{2}(C): A_{1}$ et $A_{2}$ sont des lexèmes prédicatifs antonymes qui ont tous leurs arguments en commun.

Lorsque les antonymes sont des prédicats dont tous les arguments sont communs, ils peuvent être des verbes, des adverbes ou des adjectifs.

Deux verbes antonymes dans cette configuration peuvent être simplement coordonnés comme c'est le cas dans l'énoncé (6) ou être constitutifs d’une structure Que X ou que Y. C’est le cas dans l'énoncé suivant.

(8) «Qu'elle l'accable ou qu'elle le libère, l'ancien premier ministre et adversaire déclaré de Nicolas Sarkozy ne manquera pas de transformer cette décision en moment politique. » (Le Monde 28/05/2011, « L'avenir politique de M. de Villepin suspendu à la décision de la cour d'appel », Pascale Robert-Diard).

Claude Muller analyse une structure comparable à celle-ci dans la phrase Qu'il pleuve ou pas, je sortirai, construite sur la négation du prédicat introduit par que (Muller 1996 : 162). Dans cette phrase, la structure introduite par que sous-tend une hypothèse qui « ne prend pas la forme la plus courante (si $P$ ) mais celle d'une évocation par coordination disjonctive de plusieurs situations opposées » (ibid.). Cette hypothèse est associée à une assertion "présentée comme valide dans toutes les situations évoquées » (ibid.). Lorsque que introduit des prédicats antonymes, la branche négative de l'hypothèse ne prend plus la forme d'une négation grammaticale mais d'une négation lexicale constitutive du sens de l'un des antonymes. Muller analyse les constructions de l'hypothèse de type Que x ou pas dans le chapitre qu'il consacre aux subordonnées concessives extensionnelles de type quel(le) que soit $x$ (Muller 1996: 161-184). Ce rapprochement entre les constructions Que x ou pas et quel(le) que soit X nous semble confirmé par le fait que toutes les constructions de type que $X$ ou que $Y$, lorsqu'elles contiennent des antonymes, peuvent être paraphrasées par une concessive extensionnelle dans laquelle les antonymes sont remplacés par un lexème ou un ensemble de lexèmes qui dénote(nt) la dimension sur laquelle ils s'opposent. Ainsi, dans l'énoncé (8), la construction qu'elle l'accable ou qu'elle le libère peut être paraphrasée par quelle que soit sa teneur. La coprésence antonymique permet donc d'exprimer que l'action dénotée par transformer aura lieu quelle que soit la teneur de la décision (elle) de la Cour d’appel par rapport à Dominique de Villepin (premier ministre, l', le). On peut dès lors considérer que les concessives extensionnelles sont sémantiquement équivalentes aux constructions de type que $X$ ou que $Y$, qui sous-tendent une hypothèse. Pour distinguer cette hypothèse, décrite par Muller, de l'hypothèse classique introduite par si, nous lui donnons le nom d'hypothèse extensionnelle.

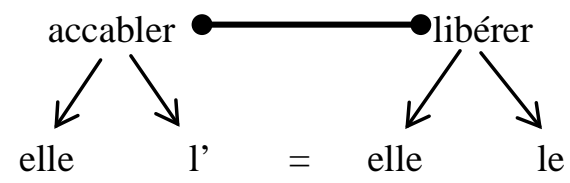

Dans l'énoncé (8), la coprésence antonymique exerce une fonction sémantico-référentielle à laquelle nous donnons le nom de fonction d'expression de l'exhaustivité. Cette fonction peut être définie sur le plan sémantique et sur le plan référentiel comme l'expression d’une totalité. Sur le plan sémantique, la coprésence antonymique dans cet énoncé permet de dénoter la totalité de la dimension sémantique sur laquelle les antonymes s’opposent. Lorsque les antonymes exercent cette fonction, leur coordination, par et / ou, est toujours paraphrasable par une concessive extensionnelle de type quel(le) que soit $X$, où $X$ représente la dimension : quelle que soit la TENEUR de la décision. Sur le plan référentiel, la dimension 
sur laquelle les antonymes s’opposent permet de définir la totalité d'une classe référentielle qui est directement attachée à cette dimension.

Lorsque les antonymes sont des adverbes, ils peuvent également exercer une fonction d'expression de l'exhaustivité, si les antonymes sont des prédicats qui opèrent sur deux autres prédicats identiques dont tous les arguments sont communs. C’est le cas dans l'énoncé (9).

(9) «Lors des précédents scrutins, le SBIH était tantôt ouvertement, tantôt discrètement allié au SDA ; cette fois, des cadres de son parti l'incitent à passer des alliances avec le SDP, désormais perçu comme le parti de l'avenir. M. Silajdzic, qui n'a pas encore annoncé ses intentions, pencherait plutôt pour un "cavalier seul", cultivant sa différence en vue des scrutins présidentiel et parlementaire, prévus pour octobre. » (Le Monde 12/04/2000, «Les électeurs des principales villes de Bosnie ont rejeté les partis nationalistes », Rémy Ourdan).

La coprésence antonymique exerce une fonction d'expression de l'exhaustivité en raison du fait que les antonymes ont le même argument, le prédicat allier : le SBIH a toujours été allié au SDA, quelle que soit la manière dont s’est manifestée cette alliance.

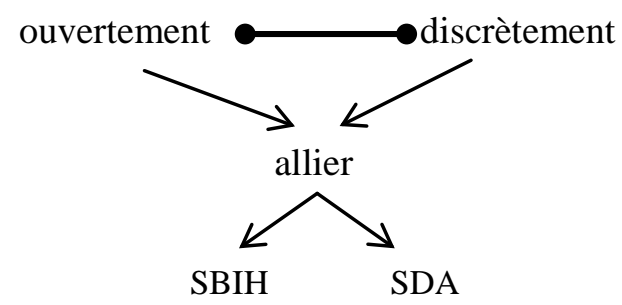

Lorsque les adjectifs antonymes ont tous leurs arguments en commun - comme l'indique le schéma cidessous dans lequel jeune et âgé peuvent être remplacés par les quatre autres paires d’adjectifs employées dans l'énoncé (1) -, ces adjectifs expriment l'exhaustivité. En première approche, on peut considérer que les antonymes fournissent simplement un principe de classement pour un même référent. En effet, en raison du fait que les antonymes permettent de constituer des subordonnées d'interrogation indirecte dépendantes du prédicat savoir, les paires antonymiques coprésentes constituent les branches d’une alternative entre lesquelles le téléphone présenté est censé pouvoir choisir pour déterminer auxquelles des douze classes référentielles, opposées deux à deux et désignées par les antonymes, son propriétaire appartient. Ces classes sont hiérarchisées ontologiquement à partir du sexe jusqu’au statut social. Malgré l'intégration des antonymes dans une alternative, l'énumération non close d'un grand nombre de paires d'antonymes nous semble indiquer que l'interprétation des coprésences antonymiques peut être l’exhaustivité : le téléphone pourra tout connaître sur son propriétaire.

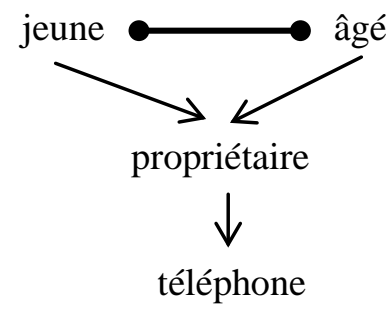

À côté des nombreux adjectifs prédicatifs antonymes employés dans cet énoncé avec le même argument, les substantifs non prédicatifs antonymes homme et femme sont employés comme arguments d'un même prédicat, comme l'indique le schéma ci-dessous. L'expression de l'exhaustivité par deux antonymes employés dans cette configuration est analysée dans le chapitre suivant. 


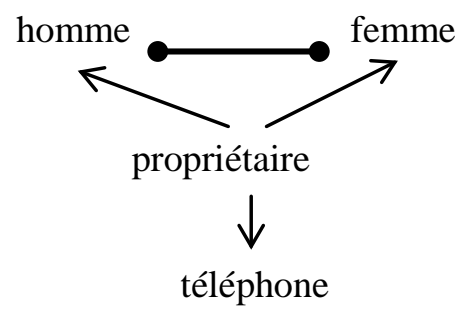

\subsubsection{Antonymes dans une fonction argumentale}

$P\left[A_{1}+A_{2}, C\right]: A_{1}$ et $A_{2}$ sont des lexèmes antonymes qui occupent la même position argumentale par rapport à un prédicat.

(10) «Tournon avait bien écrit quelques années plus tôt : "Par la seule syntaxe des langues, nous pouvons juger des vertus et des vices, de la liberté et de l'esclavage des nations." » (Le Monde 7/07/1989, « Grammaire patriotique », Denis Slakta).

Lorsque les antonymes, essentiellement des substantifs, ne sont pas des prédicats recteurs mais les arguments d'un même prédicat, ils exercent également une fonction d'expression de l'exhaustivité. Si les antonymes sont eux-mêmes des prédicats, ils ont tous leurs arguments en commun, comme dans l'énoncé (10): les antonymes ont tous nations comme argument commun. La coprésence antonymique permet d'exprimer que l'on peut juger de la qualité morale des nations et des contraintes qui pèsent sur elles.

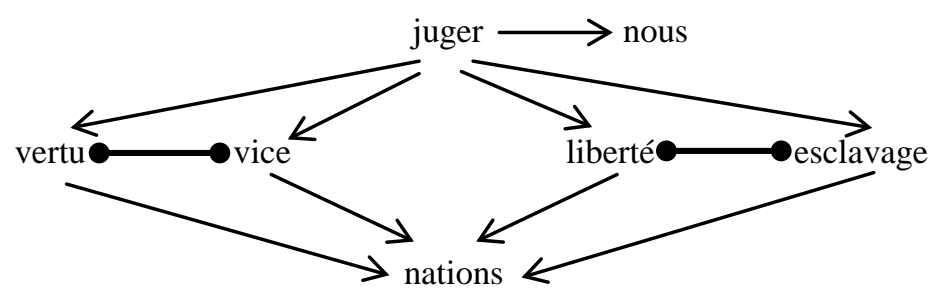

\subsubsection{Antonymes dans une fonction d'actualisation}

$\mathrm{P}_{\mathrm{A} 1}+\mathrm{A}_{2}(\mathrm{C}): \mathrm{A}_{1}$ et $\mathrm{A}_{2}$ sont des lexèmes antonymes qui exercent une fonction d'actualisation par rapport au même prédicat.

(11) «Pour la faiblesse insigne de l'interprétation, à l'exception peut-être du rôle du méchant; mais surtout pour le véritable contre sens (par rapport aux films "canoniques") que de faire de Zorro / Don Diego un bravache de jour comme de nuit, à visage découvert ou masqué.» (Le Monde 13/05/1990, "Cabou Cadin : CANAL +, 17 h 25. Duncan Reglehr et E. Lindner. Un Zorro pointé », Claude Aziza).

La coprésence de deux antonymes non prédicatifs qui exercent une fonction d'actualisation par rapport à un même prédicat exprime également l'exhaustivité. C'est le cas dans l'énoncé (11). Les substantifs jour et nuit exercent une fonction d'actualisation temporelle par rapport au même prédicat, bravache. Sont également des actualisateurs de ce prédicat la locution figée à visage découvert et l'adjectif masqué que l'on peut considérer comme des antonymes. Dans cet énoncé, les antonymes coprésents expriment l'exhaustivité : Zorro est toujours un bravache, quelle que soit son apparence.

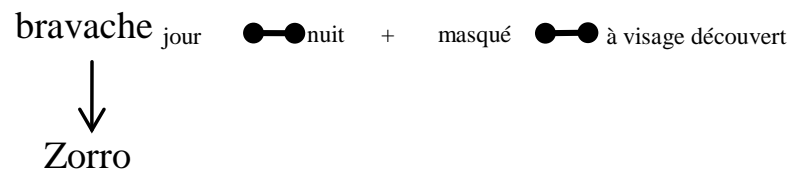




\subsection{Exhaustivité par rétroclassement}

Lorsque la coprésence antonymique, qui exprime l'exhaustivité, participe à une double mise en relation, cette fonction d'expression de l'exhaustivité s'appuie sur une autre fonction, la fonction de sousclassement.

\subsubsection{Sous-classement et rétroclassement}

Les antonymes qui exercent une fonction de sous-classement sont essentiellement des lexèmes prédicatifs qui ont pour arguments deux lexèmes désignant, en raison de la présence des antonymes, des référents différents. Dans les deux énoncés ci-dessous, les lexèmes prédicatifs antonymes sont des verbes, qui permettent de distinguer deux sous-classes de femmes (12) en fonction de ce qu'elles éprouvent par rapport à la maternité et deux sous-classes d'aliments (13) en fonction de la place qu'on leur accorde dans un régime. Cette fonction de sous-classement peut être explicitée et renforcée par l'usage de locutions comme il y a deux sortes de (12).

(12) «Eliette Abécassis, dont je ne partage pas l'approche de la maternité, dit une chose juste : il y a deux sortes de femmes, celles qui aiment à se retrouver en femelles mammifères, et celles qui détestent cela, ne veulent pas en entendre parler. » (Le Monde 13/02/2010, « Cessons d'avoir une idée unique de la gent féminine », propos d’Elisabeth Badinter recueillis par Josyane Savigneau).

(13) [à propos des aliments] « Il faut simplement en supprimer certains et en ajouter d'autres, indiquent les chercheurs. » (Le Monde 07/05/2011, « Pour se sentir mieux dans son assiette », Pascale Santi).

Dans ces configurations, la coprésence antonymique permet de distinguer, à l'intérieur de la classe référentielle à laquelle appartiennent les référents des lexèmes de la paire $B$, deux sous-classes référentielles dont ils définissent l'intension.

La division d'une classe référentielle en deux sous-classes par les antonymes est binaire et repose sur la coprésence antonymique et, éventuellement, sur des déterminants ou des pronoms qui jouent également un rôle dichotomisateur (les autres, d'autres, le reste, par exemple), mais non sur une détermination à un autre niveau. Les antonymes sont donc les seuls éléments de l'énoncé, dans lequel ils sont employés en coprésence, à fournir le principe organisateur des sous-classes qu’ils définissent.

La fonction d'expression de l'exhaustivité peut être exercée par des antonymes dont la coprésence exerce également une fonction de sous-classement. L'expression de l'exhaustivité doit alors être considérée comme la résultante d'un processus de recomposition, défini comme l'inverse du processus de division qui produit un sous-classement. Nous donnons à ce processus le nom de processus de rétroclassement. Ces deux processus inverses sont illustrés par les schémas ci-dessous.
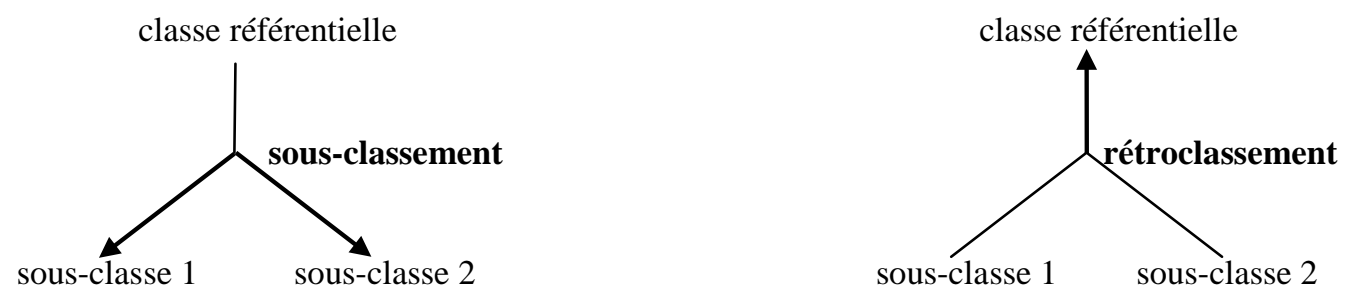


\subsubsection{Marqueurs et indices d'exhaustivité}

Comme les schémas prédicatifs dans lesquels la coprésence antonymique exprime l'exhaustivité dans une double mise en relation sont les mêmes que ceux dans lesquels elle exerce une fonction de sousclassement, des marqueurs permettant de déterminer si c'est le sous-classement ou le rétroclassement qui est le processus saillant doivent être établis. Nous avons identifié deux marqueurs syntaxiques d'exhaustivité.

(1) L’exhaustivité est explicitement sous-tendue par la présence d'un déterminant ou d'un prédéterminant qui exprime une totalité (tout, tous) et/ou par une conjonction (le plus souvent formée de que) qui introduit une hypothèse extensionnelle (qu'il soit $x$ ou y) ou par une proposition concessive extensionnelle (quel que soit) (voir 3.1.1). Cette explicitation de l'exhaustivité a pour corollaire évident l'absence d'éléments de dichotomisation explicite (d'autres, les autres, le reste).

Dans l’énoncé (14), un prédéterminant de totalité explicite l’exhaustivité.

(14) « Il fallait s'y attendre, le scélérat a encore frappé. Et tous les communicateurs, les petits et les grands, seront atteints, puisque tel est, à l'évidence, l'objet de cette nouvelle agression. » (Le Monde 05/05/1991, «Du coton sur les mots », André Laurens).

La dimension sémantique par rapport à laquelle la totalité d'une classe référentielle est définie est toujours exprimable par une concessive extensionnelle de type quel(le) que soit $x$, où $X$ représente la dimension (voir 3.1.1). Le plus souvent, cette concessive n’est pas explicitée, elle est simplement une paraphrase possible de la conjonction ou de la disjonction des antonymes, mais il peut arriver qu'elle se superpose à la coprésence antonymique comme c’est le cas dans l'énoncé (15).

(15) «Ils [les sénateurs] ont rendu obligatoire le tatouage, quel que soit le mode de cession d'un animal (gratuit ou onéreux). » (Le Monde 22/04/1989, « Un projet de loi adopté à l'unanimité au Sénat Les animaux domestiques devront être tatoués », Anne Chaussebourg).

(2) Même en l'absence d'un déterminant ou d'une conjonction explicitant l'exhaustivité, celle-ci est marquée par le fait que chacun des membres de la paire d'antonymes, ainsi que leurs éventuels arguments exprimés, exercent une fonction argumentale par rapport au même prédicat, à condition que ce prédicat ne soit pas un prédicat d'alternative comme choisir, privilégier, préférer ou se concentrer sur. Dans l'énoncé (16), les ensembles formés par les antonymes, qui sont ici des adjectifs, et leur argument exercent également une fonction actualisatrice par rappport à un même prédicat, fasciner. Les séquences grand écran et petit écran sont figées et désignent le cinéma et la télévision. La coprésence antonymique permet d’exprimer que la Mafia fascine quel que soit le média visuel, sur lequel elle apparaît.

(16) « Déjà en tête des ventes, quelques jours à peine après sa sortie, Mafia II [jeu vidéo] prouve que le monde de la Mafia continue de fasciner sur grand ou petit écran. » (Le Monde 19/09/2010, "Dans les pas de Vito, petite frappe de la mafia », Guillaume Fraissard).

À côté de ces deux marqueurs qui imposent une interprétation d'exhaustivité, on peut relever, comme indices d'exhaustivité, des caractéristiques généralement identifiables dans les énoncés où la coprésence antonymique exerce une fonction d'expression de l'exhaustivité, mais qui ne suffisent pas à elles seules à assurer l'interprétation totalisante.

(1) L'emploi des antonymes comme épithètes détachées du lexème qui est leur argument est un indice d'exhaustivité. C’est le cas dans l'énoncé (17).

(17) «Au service des urgences de Sainte-Marguerite, les malades, gravement ou légèrement atteints, doivent traverser la salle d'attente des familles et ils sont souvent transférés des brancards des pompiers à ceux de l'hôpital, sous les regards 
de tous. »(Le Monde15/09/1999, «Les personnels des services des urgences marseillais mènent une grève pour protester contre l'insécurité », Michel Samson).

(2) Le fait que les antonymes fassent partie d'une liste peut être un indice d'exhaustivité. Un même énoncé peut ainsi contenir plusieurs paires d'antonymes dont les membres sont coordonnés l'un à l'autre et qui font partie d'une énumération. L'emploi de l'adjectif inépuisable renforce cette énumération (18).

(18) « Dans ce désert plein de dangers, un certain Kader Bel Kader est, si l'on peut dire, comme un poisson dans l'eau. Ce Robin des sables, bien équipé, vend de la protection à grande échelle. Il fournit des gardes du corps, des vivres, des cigarettes de contrebande, des appareils électroniques et naturellement des armes de tout acabit. "Il avait surtout une inépuisable provision d'histoires, longues ou courtes, anciennes ou récentes, vraies ou fausses. Grâce à elles, le désert devenait un village." » (Le Monde 30/04/2010, « Fiction diplomatique », Robert Solé).

(3) Les arguments éventuels non identiques des antonymes et/ou des substantifs qui en dépendent restent non exprimés, ce qui permet à l'énoncé de conserver un haut niveau de généralité. C’est le cas dans l’énoncé (19), où l’absence de déterminant participe également à cet effet de généralité.

(19) «Dans son nouveau livre, La République, la pantoufle et les petits lapins (éd. Desclée de Brouwer, 150 p., 17,90 euros), André Glucksmann revendique l'athéisme en politique : liberté d'approuver, liberté de contester. » (Le Monde 13/05/2011, «Sarkozy et les intellectuels ; La rupture », Marion Van Renterghem).

Les antonymes exercent une fonction argumentale par rapport aux deux occurrences du prédicat liberté. Les arguments agents de chacune de ces occurrences et les arguments agents et patients des antonymes approuver/contester ne sont pas exprimés. L'on peut toutefois supposer que l'agent de ces trois prédicats pourrait être n'importe quel être humain et le patient des antonymes n’importe quelle décision politique.

Cette indétermination des arguments, potentiellement différents, jointe à la construction parallèle et énumérative sans relateur, permet à la coprésence antonymique de dépasser sa fonction de sousclassement pour exprimer l'exhaustivité : l'athéisme politique permet toute liberté en regard de la dimension EXPRIMER UN AVIS.

Si deux agents ou patients non communs aux deux occurrences de liberté et aux deux antonymes avaient été exprimés, la coprésence antonymique aurait permis de les mettre en contraste (voir 2.1 et 2.3). C’est le cas dans des exemples forgés tels que Tu as la liberté d'approuver, moi j'ai la liberté de contester ou J'ai la liberté d'approuver les décisions du gouvernement et j'ai la liberté de contester tes prises de position.

Les marqueurs et les indices que nous venons d’identifier permettent, dans la plupart des énoncés, de distinguer les cas où la coprésence antonymique exerce une fonction de sous-classement et les cas où ce sous-classement est subsumé par une expression de l'exhaustivité.

\section{Conclusions}

L’expression de l'exhaustivité a la particularité de faire partie des fonctions exercées par des antonymes qui participent à une simple mise en relation (Qu'elle l'accable ou qu'elle le libère, il tirera parti de la décision) et des fonctions exercées par des antonymes qui participent à une double mise en relation (Tous les communicateurs, les petits et les grands, seront atteints). Malgré une finalité commune - l'expression d'une totalité - qui leur permet de constituer deux faces d'une même fonction, nous avons choisi de distinguer l'exhaustivité sans rétroclassement (simple mise en relation) et l'exhaustivité par rétroclassement (double mise en relation), car elles nous sont apparues comme le résultat de deux mécanismes très différents. Ces mécanismes sont plus complexes que la coordination de surface $X$ et/ou $Y$, à laquelle Jones (2002) et Jones et al. (2012) associent l'exhaustiveness, parce qu'ils se situent à un niveau plus profond de structuration de l'énoncé, le niveau des relations prédicat-arguments. Ce niveau met en jeu la combinatoire des antonymes qui dépend de la partie du discours à laquelle ils appartiennent. 
Lorsque tous les arguments des antonymes sont co-référentiels, et ne sont le plus souvent exprimés qu'une fois, lorsque les antonymes sont des arguments de même rang ou des actualisateurs d'un même prédicat, la coprésence antonymique exerce une fonction d'expression de l'exhaustivité dans une simple mise en relation (Qu'elle l'accable ou qu'elle le libère, il tirera parti de la décision). Aucune paire B ne peut être identifiée.

Lorsque les antonymes ont pour arguments/actualisateurs des lexèmes qui désignent des référents différents appartenant à une même classe, la coprésence antonymique exerce une fonction de sousclassement (Il faut supprimer certains aliments et en ajouter d'autres) ou une fonction d'expression de l'exhaustivité par rétroclassement (Tous les communicateurs, les petits et les grands, seront atteints) au sein d'une double mise en relation. Même en l'absence d'un déterminant ou d'une conjonction explicitant l'exhaustivité, celle-ci est marquée par le fait que chacun des membres de la paire d'antonymes, ainsi que leurs éventuels arguments exprimés, exercent une fonction argumentale par rapport au même prédicat, à condition que ce prédicat ne soit pas un prédicat d'alternative comme choisir, privilégier, préférer ou se concentrer sur. Contrairement aux cas où la coprésence antonymique exerce une fonction de sousclassement, le deuxième membre de la paire B est le plus souvent sous-entendu.

Au terme de cette contribution, nous espérons avoir mis en lumière la nature sémantico-référentielle de la fonction d'expression de l'exhaustivité et la possibilité pour des paires antonymiques relevant de diverses parties de discours de l'exercer dans des configurations sémantiques et syntaxiques plus riches que ne le laisse paraître la description des chaînes syntaxiques linéaires dans lesquelles les antonymes s’insèrent.

\section{Références bibliographiques}

Blank, A. (2000). "Pour une approche cognitive du changement sémantique lexical : aspect sémasiologique », Mémoires de la Société de Linguistique de Paris, 9, 59-74.

Coseriu, E. (1975). «Vers une typologie des champs lexicaux ». Cahiers de lexicologie, 27, 30-51.

Grevisse, M. (2003). Précis de grammaire française. Louvain-la-Neuve: De Boeck/Duculot, $30^{\mathrm{e}}$ édition, $7^{\mathrm{e}}$ tirage.

Gross, G. (2012), Manuel d'analyse linguistique : approche sémantico-syntaxique du lexique. Villeneuve-d'Ascq, Presses universitaires du Septentrion.

Jones, S. (2002), Antonymy : A Corpus-based Perspective, Londres, Routledge.

Jones, S., Murphy, L., Paradis, C., Willners, C. (2012). Antonyms in English. Construals, Constructions and Canonicity. Cambridge: Cambridge University Press.

Mayaffre, D. (2008). « De l'occurrence à l'isotopie. Les co-occurrences en lexicométrie », Syntaxe et sémantique, 9, 53-72.

Mejri, S. (2011), « Présentation de la discussion sur le figement linguistique et les trois fonctions primaires (prédicats, arguments, actualisateurs) », Neophilologica, 23, 9-14.

Mettinger A. (1994), Aspects of Semantic Opposition in English, Oxford, Clarendon Press.

Morlane-Hondère, F. (2008). Combiner proximité distributionnelle et patrons linguistiques pour repérer l'antonymie, Mémoire de Master 1 en collaboration avec Marjorie Raufast. Université de Toulouse II - Le Mirail. Publication en ligne : http://w3.erss.univ-tlse2.fr/textes/pagespersos/morlane/index.html

Muller, C. (1996), La subordination en français : le schème corrélatif. Paris, A. Colin. 